\title{
Information Can Wreck Cooperation: A Counterpoint to Kandori (1992)
}

\author{
Yuichiro Kamada ${ }^{a, *}$, Scott Duke Kominers ${ }^{a, b}$ \\ ${ }^{a}$ Department of Economics, Harvard University, Cambridge, MA 02138, USA \\ ${ }^{b}$ Harvard Business School, Cambridge, MA 02138, USA
}

\begin{abstract}
We propose a simple model of repeated games with private monitoring and time-varying information structures. We then obtain an example demonstrating that the set of achievable equilibrium payoffs may shrink when players' information regarding opponents' information structures is increased.
\end{abstract}

Keywords: correlated signal structure, prisoner's dilemma, private monitoring, repeated game

JEL: C72, C73, D82

\section{Introduction}

It is well-known that repeated interaction among economic agents may lead to sustained cooperation, even when the agents would not cooperate in a one-shot relationship. ${ }^{1}$ Such results may arise even if monitoring is "imperfect" - that is, if agents cannot perfectly observe the opponents' actions. $^{2}$

Formalizations of imperfect monitoring typically assume that agents receive signals about their opponents' actions, and that these signals follow

\footnotetext{
The authors are grateful to Drew Fudenberg, Sonia Jaffe, Michihiro Kandori, Yuichi Yamamoto, and Xiaoqi Zhu for helpful comments. The second author was partly supported by a National Science Foundation Graduate Research Fellowship.

*Correponding author. Littauer Center, Harvard University, 1805 Cambridge Street, Cambridge, MA 02138, USA. Tel.: +1 857 9988668; fax: +81 458315540.

Email addresses: ykamada@fas.harvard.edu (Yuichiro Kamada), kominers@fas.harvard.edu (Scott Duke Kominers)

${ }^{1}$ For example, see Fudenberg and Maskin (1986).

${ }^{2}$ See Abreu et al. (1990) and Fudenberg et al. (1994).
} 
publicly known probability distributions, the so-called signal structure. Typically, it is additionally presumed that the signal structure is invariant over time.

But in many situations of interest, the signaling structure does exhibit change over time. In such a setting, it is natural to assume that players' signal structures may be distinct, but that the precision of the signal received by one player is not completely independent of the precision of the signals received by opponents.

For example, suppose that two competing firms can observe each other with high probability by looking out the windows if it is sunny, but only with low probability if it is foggy. In this setting, a firm can estimate the likelihood that its production level was observed, given its degree of success in observing competitors' production levels. ${ }^{3}$

In Section 2, we introduce a simple model of time-varying signal structures which captures the notion of correlated signal precision. We then examine time-varying signal structures in a prisoner's dilemma game, demonstrating in Section 3 that the set of achievable equilibrium payoffs may shrink when players know more about their opponents' information structures.

Our main example is a counterpoint to the results of Kandori (1992) for the effects of signal structure precision upon equilibria in games of imperfect monitoring. Specifically, while Kandori (1992) shows that "more precise" 4 signals result in "more equilibria," ${ }^{5}$ we demonstrate that "increased information" alone need not yield additional equilibria. Indeed, in our setting, the

\footnotetext{
${ }^{3}$ In the example just described, the correlation between the firms' signaling structures is positive. This correlation might also be negative, as the following (slightly stylized) example shows: Two rival firms have agreed to coordinate on a new product campaign, but there is only one person, "person $X$," who serves on both firms' boards. The board meetings are held concurrently, so that if $X$ attends part of one then he is less likely to attend the other. But everyone is focused on work while the meetings are active, so that nobody takes note of when people enter and exit. And $X$ sometimes slacks off-attending neither meeting-so that it is not to his advantage to reveal to either firm which meetings he has actually attended. Each day, $X$ reports in to each firm $i$ anything he was able to observe from the meeting of firm $-i$ that day. Given whether $X$ was able to observe $-i$, the fixed likelihood that $X$ slacks off, and the distance between the two firms, firm $i$ can estimate the likelihood that $X$ attended part of firm $i$ 's meeting.

${ }^{4}$ Kandori (1992) measures the precision of signal structures using the concept of "garbling." For definitions and details on this concept, see Blackwell and Girshick (1954).

${ }^{5}$ This measurement is conducted in the sense of set inclusion.
} 
set of equilibrium payoffs may be decreasing in the amount of information that player $i$ has regarding player $-i$ 's information structure. ${ }^{6}$

Although our example represents a corner case, we believe that the insight provided applies to more general settings. The objectives of this note are to generate attention to the setting and to provide a simple example where the correlation of signal structure yields a somewhat counterintuitive consequence.

\section{The Model}

There are two players, 1 and 2 . They engage in a repeated interaction with infinite horizon and discrete time, $t=1,2, \ldots$ In each stage, a simultaneousmove stage game with the following payoff matrix is played:

\begin{tabular}{c|cc} 
& $C_{2}$ & $D_{2}$ \\
\hline$C_{1}$ & $(1,1)$ & $(-h, 1+d)$ \\
$D_{1}$ & $(1+d,-h)$ & $(0,0)$.
\end{tabular}

Here, player 1 chooses a row $\left(C_{1}\right.$ or $\left.D_{1}\right)$ and player 2 chooses a column $\left(C_{2}\right.$ or $\left.D_{2}\right)$. We assume that $d, h>0$ so that the stage game is a prisoner's dilemma. Additionally, we assume $-1<d-h<1$, so that the sum of payoffs is maximized at $(C, C)$, and minimized at $(D, D)$.

At each stage, each player $i$ is in one of two states: $O_{i}$ or $N_{i}$. In state $O_{i}$, player $i$ observes player $-i$ 's action perfectly, whereas in state $N_{i}$, he does not observe anything.

The objective of player $i$ is to maximize the discounted sum of payoffs given by

$$
\sum_{t=1}^{\infty} \delta^{t-1} u_{i}\left(a_{i, t}, a_{-i, t}\right),
$$

where $\delta \in(0,1)$ is a discount factor, $a_{i, t}$ is player $i$ 's action in stage $t$, and $u_{i}\left(a, a^{\prime}\right)$ is player $i$ 's stage game payoff when he takes action $a$ and the opponent takes action $a^{\prime}$.

\footnotetext{
${ }^{6}$ Somewhat similarly, Kandori and Obara (2006) present an example in which decreased sensitivity of signals to actions leads to more efficient (limit) equilibria. However, the model and logic behind the "less is more" result of Kandori and Obara (2006) are quite different from those driving our results.
} 
There is a joint probability distribution $\pi$ over the set

$$
\left\{\left(O_{1}, O_{2}\right),\left(O_{1}, N_{2}\right),\left(N_{1}, O_{2}\right),\left(N_{1}, N_{2}\right)\right\}
$$

of possible states of the world. For simplicity we assume that

$$
\pi\left(\left\{\left(O_{1}, O_{2}\right),\left(O_{1}, N_{2}\right)\right\}\right)=\pi\left(\left\{\left(O_{1}, O_{2}\right),\left(N_{1}, O_{2}\right)\right\}\right)=\frac{1}{2} \cdot 7
$$

We then let $p$ be the conditional probability that player 2 observes player 1 's action given that player 1 observes player 2's action, so that

$$
\pi\left(\left\{\left(O_{1}, O_{2}\right)\right\}\right)=\frac{p}{2}
$$

If $p=0$, then the information structures are perfectly negatively correlated; if $p=\frac{1}{2}$, then they are independent; and if $p=1$, then they are are perfectly positively correlated. ${ }^{8}$

A strategy of player $i$ is just a map from the set of possible histories to a probability distribution over actions. We analyze sequential equilibria of this game, which we simply call "equlibria" in the sequel.

\section{Analysis}

We now describe two key results obtained in our setting. Since all the proofs are straightforward, we omit them.

\section{Proposition 1.}

1. If $p \in(0,1)$, then there exists $\bar{\delta} \in(0,1)$ such that, for any $\delta \in[\bar{\delta}, 1)$, the expected payoff of full cooperation, $\frac{1}{1-\delta}$, can be achieved in equilibrium.

2. If $p=0$, then there exists $\bar{\delta} \in(0,1)$ such that, for any $\delta \in[\bar{\delta}, 1)$, the best expected payoff achievable in equilibrium is $\frac{1+d-h}{2(1-\delta)}$.

3. If $p=1$, then there exists $\bar{\delta} \in(0,1)$ such that, for any $\delta \in[\bar{\delta}, 1)$, the best expected payoff achievable in equilibrium is $\frac{1}{2(1-\delta)}$.

\footnotetext{
${ }^{7}$ We believe relaxation of this assumption to be straightforward.

${ }^{8}$ Consequently, if $p=\frac{1}{2}$, then each player has no information about the opponent's information structure, while as $p$ approaches 0 or 1, players' information about opponent's information structures increases.
} 
This proposition shows that, in almost all cases, full cooperation can be achieved when players are sufficiently patient. ${ }^{9}$ Surprisingly, however, it is no longer possible to sustain cooperation when the correlation between signal structures is perfect. The intuition for this latter fact is clear: if a player is certain that his actions will not be observed, then he has no incentive to cooperate.

In many settings of economic interest, the discount factor is not very high. ${ }^{10}$ It is therefore important to know the minimal value $\delta^{*}$ at which the expected payoff of full cooperation can be achieved. ${ }^{11}$ Setting

$$
r=\max \{p, 1-p\}
$$

we may compute $\delta^{*}$ explicitly.

Proposition 2. $\delta^{*}=d /(d+1-r)$ holds.

Note that the value of $\delta^{*}$ obtained in Proposition 2 is consistent with Parts 2 and 3 of Proposition 1. If $p \in\{0,1\}$, then we have $r=1$, so $\delta^{*}=1$. But by assumption, we have $\delta<1$, so cooperation cannot be sustained when $p \in\{0,1\}$.

We now observe two interesting comparative statics of $\delta^{*}$. The first is standard: $\delta^{*}$ is increasing in $d$, hence it is increasingly difficult to sustain cooperation as the "temptation" $d$ increases.

The second comparative static is our main observation and our counterpoint to Kandori (1992): $\delta^{*}$ is increasing in $r$. The information held by each player at each stage is increasing in the correlation between signal precision levels. Thus $r$ may be interpreted as a measurement of the amount of information that player $i$ has about player $-i$ 's information structure. ${ }^{12}$

\footnotetext{
${ }^{9}$ This result likely extends to a setting with slighly imperfect monitoring (in place of the perfect monitoring structure used in our example), in the sense that sufficiently strong signals lead to near-cooperation when players are sufficiently patient. (Sekiguchi (1997) presents a result along these lines.) Also, the result can be easily extendable to the case where the states are serially correlated with Markov process.

${ }^{10} \mathrm{Or}$, equivalently, players' interactions are not very frequent.

${ }^{11}$ For $p \in(0,1)$, this is equivalent to saying that $\delta^{*}$ is the minimal value of $\bar{\delta}$ in Part 1 of Proposition 1.

${ }^{12}$ This value $r$ achieves its minimum $\frac{1}{2}$ when the information structure is independent, and increases as the correlation increases (positively or negatively), to a maximum 1 achieved when the information structure is perfectly correlated.
} 
Hence, the comparative static in $r$ indicates that cooperation becomes more and more difficult to sustain as players' information about their opponents' signals is increased.

Note that this example does not contradict the main result of Kandori (1992), since the notion of "more information" in our model differs from that Kandori uses. Nonetheless, our results indicate that the type of "information precision" under investigation significantly impacts the effects of increased precision upon the set of achievable equlibria.

\section{References}

Abreu, D., Pearce, D., Stachetti, E., 1990. Towards a theory of discounted repeated games with imperfect monitoring. Econometrica 58, 1041-1064.

Blackwell, D., Girshick, M. A., 1954. Theory of Games and Statistical Decisions. Wiley.

Fudenberg, D., Levine, D., Maskin, E., 1994. The folk theorem in repeated games with imperfect public information. Econometrica 62, 997-1039.

Fudenberg, D., Maskin, E., 1986. The folk theorem in repeated games with discounting or with incomplete information. Econometrica 54, 533-554.

Kandori, M., 1992. The use of information in repeated games with imperfect monitoring. Review of Economic Studies 59, 581-594.

Kandori, M., Obara, I., 2006. Less is more: an observability paradox in repeated games. International Journal of Game Theory 34, 475-493.

Sekiguchi, T., 1997. Efficiency in repeated prisoner's dilemma with private monitoring. Journal of Economic Theory 76, 345-361. 